\title{
Editorial \\ More intrinsic parameters should be used in assessing degeneration of articular cartilage with quantitative ultrasound
} Yong-Ping Zheng ${ }^{1,2}$ and Yan-Ping Huang ${ }^{1}$

\author{
1'Department of Health Technology and Informatics, The Hong Kong Polytechnic University, Kowloon, Hong Kong, China \\ ${ }^{2}$ Research Institute of Innovative Products and Technologies, The Hong Kong Polytechnic University, Kowloon, Hong Kong, China
}

Corresponding author: Yong-Ping Zheng, ypzheng@ieee.org

Published: 16 December 2008

This article is online at http://arthritis-research.com/content/10/6/125

Arthritis Research \& Therapy 2008, 10:125 (doi:10.1186/ar2566)

c) 2008 BioMed Central Ltd

See related research by Kuroki et al., http://arthritis-research.com/content/10/4/R78

\begin{abstract}
During the last decade, the quantitative ultrasound technique has been widely employed as a versatile modality to investigate a thin but crucial tissue layer - the articular cartilage. Previous studies provide information about the morphology and mechanical and acoustic properties of the tissue derived from ultrasound measurements and correlate them with cartilage degeneration. In a previous issue of Arthritis Research \& Therapy, Kuroki and colleagues presented a study about the relationship between International Cartilage Repair Society grading and ultrasound echo magnitude, duration, and interval in human knee cartilage. We think indirect measurements of the intrinsic physical characteristics of cartilage, as reported in this study, should be interpreted more carefully as they can be affected by many experimental and physical factors. In this editorial, we offer our opinion that more intrinsic material parameters should be selected for the assessment of degeneration states of cartilage using quantitative ultrasound.
\end{abstract}

It has been widely reported that the early degeneration of articular cartilage involves the loss of proteoglycan and damage of collagen matrix integrity, which together may lead to tissue softening, swelling, thickness changing and roughening of the cartilage surface. During the last decade, researchers have made many efforts to correlate these changes reflected by mechanical, acoustical, and morphological parameters of cartilage with its degeneration levels using ultrasound and other techniques. In comparison with conventional ultrasonography for imaging joints, quantitative ultrasound measurements using higher frequencies not only achieve better resolution for thickness and surface roughness but also provide acoustic and mechanical parameters of articular cartilage for early diagnosis of degeneration. Since quantitative ultrasound probes are normally designed to use with arthroscopy, it is able to access any location of a joint with a cost of being minimally invasive. In this editorial, we limited our discussion specifically to quantitative ultrasound measurement in relation to the work reported by Kuroki and colleagues [1] in a previous issue of Arthritis Research \& Therapy. For details of conventional noninvasive ultrasonography for osteoarthritis evaluation, readers may refer to recent review articles [2-4].

It is encouraging to see the development of quantitative ultrasound techniques from laboratory to clinical use for the assessment of articular cartilage degeneration. In their recent study, Kuroki and colleagues [1] investigated the correlations between International Cartilage Repair Society (ICRS) grading and ultrasound signal intensity, echo duration, and interval between signals. It was reported that 'signal intensity of grade 0 cartilage was significantly greater than those of grade 1 , grade 2, or grade 3 cartilage'. The result appears to suggest that 'signal intensity' alone could potentially be used to differentiate normal cartilage from that with degeneration provided that signal intensity information of the normal state is available. In our opinion, such potentials should be considered carefully, taking into account the ultrasound measurement system and the intrinsic physical properties of the tissue.

The 'signal intensity, echo duration, and interval between signals' derived from the reflected ultrasound echoes using wavelet or other methods are not intrinsic mechanical properties and can be affected by different factors $[5,6]$. In physics, the 'signal intensity' relates to the tissue reflection coefficient, impedance, elastic modulus, and surface conditions, not simply 'a measure of superficial cartilage integrity' as defined by the authors [1]. The 'echo duration' relates to frequencydependent attenuation, surface roughness, and curvature. The 'interval between signals' relates to both tissue thickness and speed of sound. Furthermore, with respect to a specific ultrasound system setup (including the parameters used for 
pulser/receiver and focusing of transducer), signal intensity is also sensitive to the distance between the transducer and tissue, orientation of the transducer (incidence angle of ultrasound beam), and curvature of the tissue surface besides the tissue state. The echo duration can be affected by the characteristics of ultrasound transducer, such as its frequency response and beam width, and those of pulser/ receiver, such as pulse duration, filter setting, and damping. Therefore, unless all affecting factors can be controlled and standardized, even a significant correlation between ultrasonic parameters and clinical grades obtained by a specific device might provide limited information.

Furthermore, this study used 'interval between signals' to correlate with ICRS grades, making the result difficult to interpret because the speed of sound and the thickness both can contribute to the change of the interval. Although the authors did discuss the effect of swelling on the speed of sound, they neglected to address the fact that thickness could also be affected during swelling. If these intrinsic physical properties are reported, comparison of results from different specimens, species, devices, and investigative approaches would become more feasible and meaningful. The making of assumptions, which are used as the basis for obtaining the intrinsic physical properties, is inevitable. Knowing what assumptions were made helps researchers to anticipate or know what possible factors may affect the results. This in turn will promote better understanding of the assumptions and factors involved, which leads to the development of new approaches.

Instead of using 'signal intensity', a number of papers have reported the use of ultrasound reflection coefficient [7], integrated reflection coefficient [8], and echo frequency spectrum [9] to assess cartilage degeneration. To provide a better thickness measurement using ultrasound, researchers have made efforts to investigate the depth dependency of speed of sound [10] and techniques for measuring speed of sound in situ [11]. To quantify the surface roughness directly, a new method using ultrasound scanning has been proposed [12]. Ultrasound has been used along with mechanical disturbance methods, such as indentation [7,13], water jet indentation [14], compression [15], or osmosis loading [16], to measure more intrinsic mechanical properties and their depth dependency. Some of these techniques may not yet be ready for clinical trials, but they can provide valuable fundamental information to drive development forward, with the ultimate aim of providing reliable clinical tools for early diagnosis of osteoarthritis. Readers may refer to Wang and colleagues [17] for a more comprehensive list of related references.

In summary, while quantitative ultrasound has demonstrated promising potential in assessing cartilage degeneration, an understanding of its physical principles and limitations is necessary. We suggest that engineers in the field aim to develop new techniques to obtain intrinsic physical para- meters of cartilage. We also hope that clinicians in the field (or through collaboration with their engineer colleagues) fully understand the physical principles of the measurement and what the obtained parameters physically represent before trying to interpret the data and look for correlations. The success of quantitative ultrasound for bone mineral density assessment should be attributed in part to its direct measurement of intrinsic physical parameters, including speed of sound and attenuation [18], which made crosscenter comparison and instrumentation standardization possible. We believe only quantitative ultrasound assessment using more intrinsic physical parameters can lead to reliable clinical devices for early evaluation of osteoarthritis. Finally, we commend Kuroki and colleagues for demonstrating to us the use of a miniaturized probe with simplified data interpretation for clinical trials. No doubt, advancement based on this work will ensue.

\section{Competing interests}

The first author holds 3 patents that can be potentially used for the assessment of articular cartilage:

1. Zheng YP and Choi APC. Measurement of modulus and Poisson's ratio simultaneously from indentation. US Provisional Patent Application. No. 60/580,679. Chinese patent filed. 200610077789.6. Apr 2006.

2. Mak AFT and Zheng YP, Portable ultrasound palpation device. US Patent issued, No. 6494840, Dec 2002.

3. Zheng YP and $\mathrm{Lu} \mathrm{MH}$, Noncontact measurement of material properties. US patent issued. No. 7124636. Oct 242006

\section{Acknowledgments}

The works of the authors in the field of ultrasound assessment for articular cartilage were supported in part by The Hong Kong Research Grant Council (F-HK12/01T, PolyU5199/02E, PolyU5245/03E, and PolyU5318/05E) and The Hong Kong Polytechnic University (J-BB69).

\section{References}

1. Kuroki H, Nakagawa Y, Mori K, Kobayashi M, Yasura K, Okamoto Y, Suzuki T, Nishitani K, Nakamura T: Ultrasound properties of articular cartilage in the tibio-femoral joint in knee osteoarthritis: relation to clinical assessment (ICRS grade). Arthritis Res Ther 2008, 10:R78.

2. Chao J, Kalunian K: Ultrasonography in osteoarthritis: recent advances and prospects for the future. Curr Opin Rheumatol 2008, 20:560-564.

3. Moller I, Bong D, Naredo E, Filippucci E, Carrasco I, Moragues C, lagnocco $A$ : Ultrasound in the study and monitoring of osteoarthritis. Osteoarthritis Cartilage 2008, 16 Suppl 3:S4-S7.

4. Meenagh G: Ultrasound imaging for the rheumatologist - VIII. Ultrasound imaging in osteoarthritis. Clin Exp Rheumatol 2007, 25:172-175.

5. Saarakkala S, Jurvelin JS, Zheng YP, Nieminen HJ, Toyras J: Quantitative information from ultrasound evaluation of articular cartilage should be interpreted with care. Arthroscopy 2007, 23:1137-1138.

6. Kaleva E, Saarakkala S, Toyras J, Nieminen HJ, Jurvelin JS: In vitro comparison of time-domain, frequency-domain and wavelet ultrasound parameters in diagnostics of cartilage degeneration. Ultrasound Med Bio/ 2008, 34:155-159.

7. Laasanen MS, Toyras J, Hirvonen J, Saarakkala S, Korhonen RK, Nieminen MT, Kiviranta I, Jurvelin JS: Novel mechano-acoustic technique and instrument for diagnosis of cartilage degeneration. Physiol Meas 2002, 23:491-503. 
8. Cherin E, Saied A, Laugier P, Netter P, Berger G: Evaluation of acoustical parameter sensitivity to age-related and osteoarthritic changes in articular cartilage using $50-\mathrm{MHz}$ ultrasound. Ultrasound Med Biol 1998, 24:341-354.

9. Brown CP, Hughes SW, Crawford RW, Oloyede A: Ultrasound assessment of articular cartilage: analysis of the frequency profile of reflected signals from naturally and artificially degraded samples. Connect Tissue Res 2007, 48:277-285.

10. Patil SG, Zheng YP, Shi J: Measurement of depth-dependence and anisotropy of ultrasound speed of bovine articular cartilage in vitro. Ultrasound Med Biol 2004, 30:953-963.

11. Suh JK, Youn I, Fu FH: An in situ calibration of an ultrasound transducer: a potential application for an ultrasonic indentation test of articular cartilage. J Biomech 2001, 34:1347-1353.

12. Saarakkala S, Toyras J, Hirvonen J, Laasanen MS, Lappalainen R, Jurvelin JS: Ultrasonic quantitation of superficial degradation of articular cartilage. Ultrasound Med Biol 2004, 30:783-792.

13. Zheng YP, Mak AF: An ultrasound indentation system for biomechanical properties assessment of soft tissues in vivo. IEEE Trans Biomed Eng 1996, 43:912-918.

14. Lu MH, Zheng YP, Huang QH, Ling C, Wang $Q$, Bridal L, Qin L, Mak A: Noncontact evaluation of articular cartilage degeneration using a novel ultrasound water jet indentation system. Ann Biomed Eng 2008 Nov 15. [Epub ahead of print].

15. Zheng YP, Mak AF, Lau KP, Oin L: An ultrasonic measurement for in vitro depth-dependent equilibrium strains of articular cartilage in compression. Phys Med Biol 2002, 47:3165-3180.

16. Wang $\mathrm{Q}$, Zheng YP, Leung G, Lam WL, Guo X, Lu HB, Oin L, Mak AF: Altered osmotic swelling behavior of proteoglycandepleted bovine articular cartilage using high frequency ultrasound. Phys Med Biol 2008, 53:2537-2552.

17. Wang Q, Zheng YP, Qin L, Huang QH, Lam WL, Leung G, Guo $X$, Lu HB: Ultrasonic assessment of progressive proteoglycan depletion of articular cartilage in real-time. Ultrasound Med Biol 2008, 34:1085-1092.

18. Njeh CF, Boivin CM, Langton CM: The role of ultrasound in the assessment of osteoporosis: a review. Osteoporosis Int 1997, 7:7-22. 